\title{
Tratabilidade por Parâmetro Fixo para Largura em Árvore de Grafos Direcionados*
}

\author{
A. Karolinna Maia ${ }^{1}$, Raul Lopes ${ }^{1,2}$, Victor Campos ${ }^{1,3}$ \\ ${ }^{1}$ Departamento de Computação - Universidade Federal do Ceará (UFC) \\ Fortaleza - CE - Brazil \\ Grupo de Pesquisa ParGO \\ karolmaia@lia.ufc.br, raul.wayne@gmail.com, campos@lia.ufc.br
}

\begin{abstract}
The tree-width of an undirected graph $G$ is a measure of how similar $G$ is to a tree. An analogous definition is also known for directed graphs. For constant $k$, we improve on a known result and show an FPT algorithm that decides whether the tree-width of a directed graph $D$ is at most $3 k-2$ or if it admits a haven of order $k$.
\end{abstract}

Resumo. A largura em árvore de um grafo não-direcionado $G$ é uma medida de quão similar $G$ é de uma árvore. Uma definição análoga também é conhecida para grafos direcionados. Para k constante, nós melhoramos um resultado conhecido mostrando um algoritmo FPT, com parâmetro $k$, que decide se a largura em árvore de um grafo direcionado D é no máximo $3 k-2$ ou se D admite um refúgio de ordem $k$.

Parâmetros de largura em grafos podem ser vistos como uma estimava de quão similar um grafo é de uma estrutura típica. Muitos problemas conhecidamente difíceis podem ser eficientemente resolvidos em grafos com parâmetros de largura limitados, seja fazendo uso de técnicas clássicas de construção de algoritmos, como programação dinâmica, ou enunciando o problema em lógica monádica de segunda ordem, como afirmado pelo Teorema de Courcelle [Courcelle 1990]. O foco deste trabalho é em largura em árvore de grafos direcionados. Aplicações de algoritmos baseados em decomposição em árvore vão de problemas de alocação de frequência [Koster et al. 1999] ao problema do caixeiro viajante [Cook and Seymour 2003].

Foi mostrado que grades são estruturas bloqueadores para largura em árvore em grafos não-direcionados. Como a largura em árvore de uma grade completa $k \times k$ é $k$, a largura em árvore de um grafo não-direcionado deve ser pelo menos a ordem da maior grade nele contido como um grafo menor. [Robertson and Seymour 1986] mostraram que existe uma função computável $f: \mathbb{N} \rightarrow \mathbb{N}$ tal que todo grafo nãodirecionado de largura em árvore pelo menos $f(k)$ contém uma grade $k \times k$ como um grafo menor. Este resultado, conhecido como o Teorema da grade, é fundamental no estudo de parâmetros de largura em grafos não-direcionados. A partir dele, foi criado um campo de estudo conhecido como teoria da bidimensionalidade, introduzido

\footnotetext{
${ }^{2}$ Financiado pela CAPES.

${ }^{3}$ Parcialmente financiado pelo CNPq.

*Este trabalho foi parcialmente apoiado pelo CNPq - projeto Universal 425297/2016-0 e FUNCAP PRONEM PNE-0112-00061.01.00/16.
} 
em [Demaine et al. 2005], pelo qual os autores receberam o prêmio Nerode 2015. A teoria da bidimensionalidade foi usada como base para algoritmos aproximativos, subexponenciais, esquemas de aproximação em tempo polinomial e kernelizações para um grande número de problemas difíceis em grafos que excluem um grafo menor fixo. Estes incluem o problema de conjunto de vértices de retroalimentação, conjunto dominante, emparelhamento mínimo maximal e conjunto independente, entre outros. Para alguns exemplos, encaminhamos o leitor para [Demaine et al. 2005, Demaine and Hajiaghayi 2004, Fomin et al. 2010, Fomin et al. 2011].

Dado o sucesso alcançado no estudo de parâmetros de largura para problemas em grafos não-direcionados, não é surpresa que haja interesse em encontrar definições análogas para grafos direcionados. Em particular, algumas foram propostas para largura em árvore em grafos direcionados ([Johnson et al. 2001, Reed 1997], por exemplo). Um resultado análogo ao Teorema da grade, que permaneceu como uma conjectura aberta de [Reed 1999] e [Johnson et al. 2001] por quase 20 anos, foi provado recentemente para grafos direcionados. Foi mostrado que as estruturas bloqueadoras para largura em árvore grande em grafos direcionados são grades cilíndricas [Kawarabayashi and Kreutzer 2015]. Um exemplo de grade cilíndrica de ordem 4 é dado na Figura 1. Neste trabalho consideramos as definições contidas em [Johnson et al. 2001]. Detalhamos estas abaixo para fins de completude. Deste ponto em diante, utilizamos $D$ para representar um grafo direcionado.

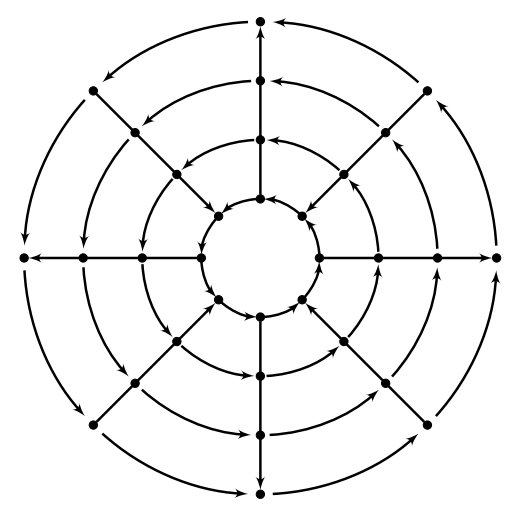

Figura 1. Grade cilíndrica de ordem 4.

Definição (Conjuntos $Z$-normais). Seja $Z$ um subconjunto de $V(D)$ e $S \subseteq V(D)-Z$. Dizemos que $S$ é $Z$-normal se não existe passeio direcionado em $D \backslash Z$ com ambas as extremidades em $S$ que utilize um vértice de $D \backslash(Z \cup S)$.

Seja $R$ uma arborescência. Para $\left\{r, r^{\prime}\right\} \subseteq V(R)$, escrevemos $r^{\prime}>r$ se $r^{\prime} \neq r$ e existe um caminho direcionado em $R$ de $r$ para $r^{\prime}$. Ademais, se $e$ é uma aresta de $R$ com cabeça $r$, escrevemos $r^{\prime}>e$ se $r^{\prime}=r$ ou $r^{\prime}>r$. Se $e$ incide em $r$, escrevemos $e \sim r$.

Definição (Decomposição arbórea). Uma decomposição arbórea de $D$ é um trio $(R, X, W)$ onde $R$ é um arborescência, $X=\left\{X_{e}: e \in E(R)\right\}$ e $W=\left\{W_{r}: r \in V(R)\right\}$ são tais que

1. $X_{e}$ e $W_{r}$ são subconjuntos de $V(D)$, para toda e $\in E(R)$ e $r \in V(R)$;

2. $\left\{W_{r}: r \in V(R)\right\}$ é uma partição de $V(D)$ em conjuntos não-vazios;

3. se e $\in E(R)$, então $\bigcup\left\{W_{r}: r \in V(R), r>e\right\}$ é $X_{e}$-normal. 
A largura de $(R, X, W)$ é o menor inteiro $k$ tal que, para todo $r \in V(R)$,

$$
\left|W_{r} \cup\left(\bigcup_{e \sim r} X_{e}\right)\right| \leq k+1 .
$$

A largura em árvore de $D$ é o menor inteiro $k$ tal que $D$ admite uma decomposição arbórea de largura $k$.

Definição (Refúgio). Um refúgio de ordem $k \mathrm{em} D$ é uma função $\beta$ atribuindo a todo conjunto de vértices $Z \subseteq V(D)$, com $|Z| \leq k-1$, o conjunto de vértices $\beta(Z)$ de uma componente fortemente conexa de $D \backslash Z$ de tal forma que se $Z^{\prime} \subseteq Z \subseteq V(D)$, então $\beta(Z) \subseteq \beta\left(Z^{\prime}\right)$.

Em [Johnson et al. 2001] foi mostrada uma relação min-max entre refúgios e decomposições em árvore de grafos não-direcionados.

Teorema 1. Seja $G$ um grafo não-direcionado e $k \geq 0$ um inteiro. Então $G$ admite um refúgio de ordem $k$ se e somente se $G$ tem largura em árvore maior ou igual a $k-1$.

Para o caso direcionado, sabemos que apenas a condição necessária do Teorema 1 apresenta uma cota inferior tão justa quanto no caso nãodirecionado [Johnson et al. 2001]. Para a suficiência, um resultado aproximativo é dado também em [Johnson et al. 2001].

Teorema 2. Seja D um grafo direcionado e $k$ um inteiro não-negativo. Existe um algoritmo polinomial, para $k$ constante, que decide se $D$ tem largura em árvore no máximo $3 k-2$ ou admite um refúgio de ordem $k$.

Adaptando o algoritmo do Teorema 2 com técnicas baseadas em separadores introduzidas em [Chen et al. 2007, Chitnis et al. 2011, Erbacher et al. 2014], melhoramos este resultado mostrando que o problema é tratável por parâmetro fixo com parâmetro $k$. Teorema 3 (Resultado principal). Seja $D$ um grafo direcionado e $k$ um inteiro nãonegativo. Existe um algoritmo FPT com parâmetro $k$ que decide se $D$ tem largura em árvore no máximo $3 k-2$ ou admite um refúgio de ordem $k$.

\section{Referências}

Chen, J., Liu, Y., and Lu, S. (2007). Directed feedback vertex set problem is fpt. In Structure Theory and FPT Algorithmics for Graphs, Digraphs and Hypergraphs, number 07281 in Dagstuhl Seminar Proceedings. Internationales Begegnungs- und Forschungszentrum für Informatik (IBFI), Schloss Dagstuhl, Germany.

Chitnis, R., Hajiaghayi, M., and Marx, D. (2011). Fixed-parameter tractability of directed multiway cut parameterized by the size of the cutset. abs/1110.0259.

Cook, W. and Seymour, P. (2003). Tour merging via branch-decompositions. J. on Computing, 15:233-248.

Courcelle, B. (1990). The monadic second-order logic of graphs. i. recognizable sets of finite graphs. Information and Computation, 85(1):12 - 75 .

Demaine, D., Fomin, V., Hajiaghayi, M., and Thilikos, M. (2005). Subexponential parameterized algorithms on bounded-genus graphs and h-minor-free graphs. J. ACM, 52(6):866-893.

Demaine, E. and Hajiaghayi, M. (2004). Fast algorithms for hard graph problems: Bidimensionality, minors, and local treewidth. Graph drawing, pages 517-533. 
Erbacher, R., Jaeger, T., Talele, N., and Teutsch, J. (2014). Directed multicut with linearly ordered terminals. CoRR, abs/1407.7498.

Fomin, F., Lokshtanov, D., Raman, V., and Surabh, S. (2011). Bidimensionality and eptas. ACM-SIAM Symposium on discrete algorithms (SODA), pages 748-759.

Fomin, F., Lokshtanov, D., Surabh, S., and Thilikos, D. (2010). Bidimensionality and kernels. ACM-SIAM Symposium on discrete algorithms (SODA), pages 503-510.

Johnson, T., Robertson, N., Seymour, P., and Thomas, R. (2001). Directed tree-width. Journal of combinatorial theory, series B, 82(01):138-154.

Kawarabayashi, K. and Kreutzer, S. (2015). The directed grid theorem. In Proceedings of the Forty-seventh Annual ACM Symposium on Theory of Computing, STOC '15, pages 655-664, New York, NY, USA. ACM.

Koster, A., van Hoesel, S., and Kolen, A. (1999). Solving frequency assignment problems via tree-decomposition. Broersma, H.J. et al 6th Twente workshop on graphs and combinatorial optimization. Univ. of Twente, Enschede, Netherlands, 3.

Reed, B. (1997). Tree width and tangles: A new connectivity measure and some applications. R. Bailey, editor, Surveys in Combinatorics, pages 87-162.

Reed, B. (1999). Introducing directed tree-width. Electronic notes in discrete mathematics, 03:222-229.

Robertson, N. and Seymour, P. (1986). Graph minors v. excluding a planar graph. Journal of combinatorial theory, series B, 41(01):92-114. 